
internationales

vol. 20 - $n^{\circ} 2 \mid 2004$

L'asile politique en Europe depuis l'entre-deux-guerres

\title{
Cartographier la demande d'asile en France. Tendances nationales, représentations départementales et réalités locales
}

Emmanuelle Bonerandi, Frédérique Bourgeois et Xavier Richard

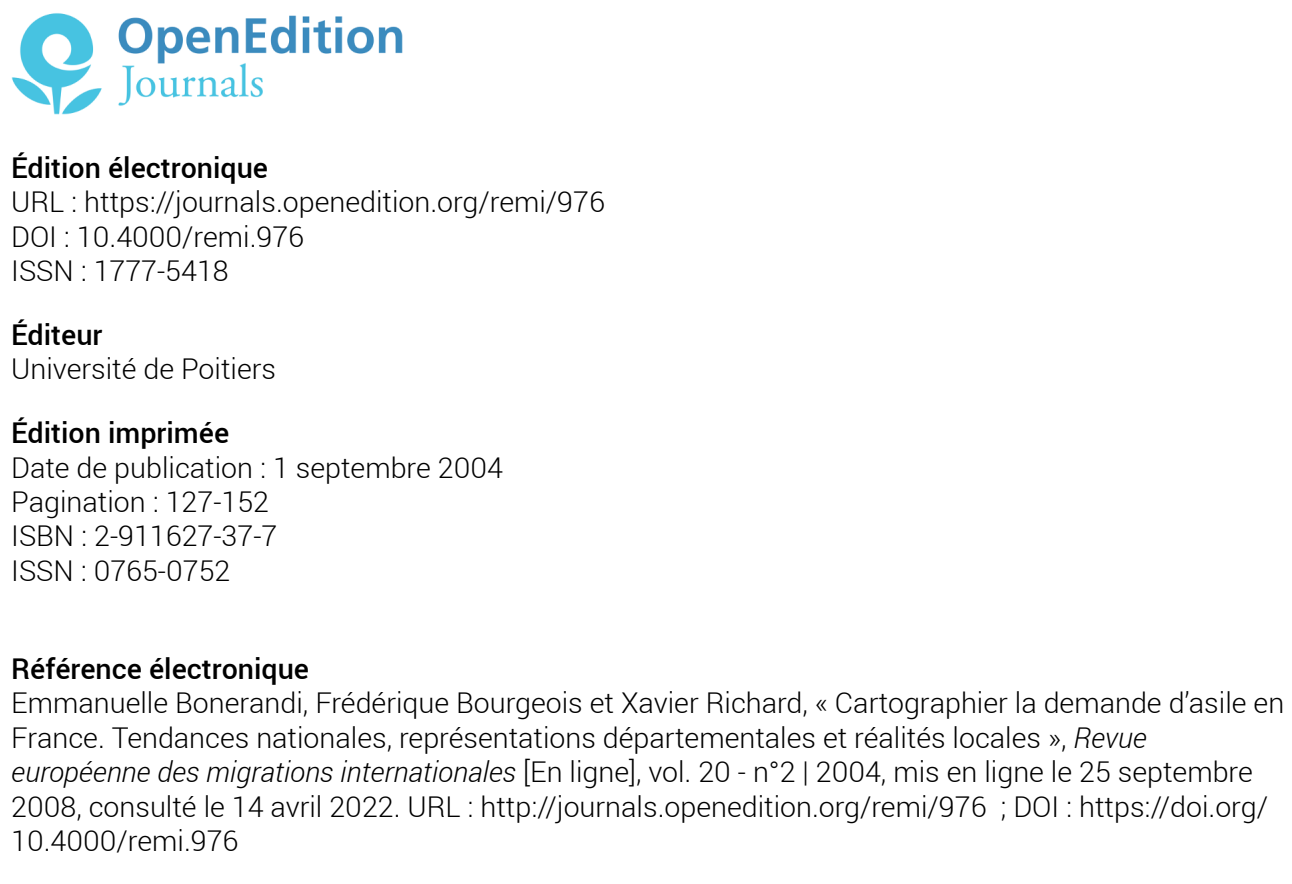

Ce document a été généré automatiquement le 14 avril 2022.

(C) Université de Poitiers 


\title{
Cartographier la demande d'asile en France. Tendances nationales, représentations départementales et réalités locales
}

\author{
Emmanuelle Bonerandi, Frédérique Bourgeois et Xavier Richard
}

\section{NOTE DE L'AUTEUR}

Avec la participation de Luc Merchez, UMR Géographie-Cités - centre Géophile, pour la réalisation des cartes

1 L'étude de la demande d'asile s'inscrit généralement dans celle des migrations internationales. Même si les phénomènes géographiques en jeu sont en partie similaires dans le fait de traverser des frontières internationales, la spécificité juridique de la procédure d'asile, relevant d'une démarche particulière de demande de protection, encadrée notamment par la convention de Genève relative au statut des réfugiés de 1951, nécessite de distinguer asile et migration pour ne pas entretenir un amalgame dangereux ${ }^{1}$.

\section{Demande d'asile et assistance : des liaisons dangereuses?}

2 La représentation cartographique de la demande d'asile, quand elle existe, fait une large place aux flux entre pays de départ et pays d'arrivée. Outre la force du modèle spatial du trait fixe représentant le flux, les données disponibles relatives à la demande d'asile sont généralement diffusées par pays d'origine et d'arrivée, notamment dans les rapports d'activité de l'OFPRA (Office Française de Protection des Réfugiés et Apatrides), organisme placé sous la tutelle du Ministère des Affaires étrangères et 
chargé de l'examen des dossiers ${ }^{2}$. Si la géographie des migrations internationales est aujourd'hui bien installée ${ }^{3}$, l'étude géographique de l'asile est un terrain encore peu défriché, la parole scientifique étant essentiellement celle des juristes, des politologues ou des sociologues ${ }^{4}$. Plus généralement, le recours au support cartographique est en outre peu développé, tant dans le domaine scientifique qu'institutionnel ou associatif.

3 À partir de l'étude du cas français, cet article entend pallier le déficit de représentations d'une question centrale en sciences sociales. La traduction cartographique des données spatialisées relatives à la demande d'asile offre une première géographie de la demande d'asile en France, entre zones de forte densité et zones potentiellement moins chargées. Elle propose une première présentation des tendances nationales, revient sur leurs expressions départementales et interroge les réalités locales.

4 Nous avons choisi de travailler à partir de deux sources d'informations fournies au niveau national concernant la demande d'asile et les hébergements qui lui sont dédiés. Cela permet de proposer deux niveaux d'analyse: d'une part, une géographie de la demande d'asile par département; d'autre part, une géographie du poids de la demande d'asile en croisant les données de demande d'asile avec les capacités d'hébergement. On peut ainsi définir une géographie prioritaire de la demande d'asile en France. Ces premiers travaux s'inscrivent dans une recherche sur l'inscription territoriale de la demande d'asile et sa déclinaison par l'entrée plus spécifique de l'hébergement.

5 Mais la carte ne dit pas tout. Nous montrerons les limites de la représentation cartographique qui, si elle présente des différenciations spatiales à différentes échelles, n'exprime que partiellement l'inscription territoriale du demandeur d'asile et les tensions d'un sujet sensible trop souvent hâtivement assimilé à celui de l'immigration.

\section{Éléments de contexte}

\section{La France, premier pays européen pour l'accueil des demandeurs d'asile en 2003}

6 Les pays de l'Union européenne ont enregistré une baisse importante des arrivées de demandeurs d'asile en 2003 (-22\% pour l'Europe des Quinze), sans commune mesure avec celle de l'année précédente. Cette situation est en grande partie le résultat des différentes dispositions mises en place dans le cadre des politiques de maittrise des flux migratoires visant à accroître le contrôle aux frontières pour restreindre les entrées sur le territoire de l'Union. La France se singularise de ses voisins européens avec des arrivées en très légère hausse $(+1,3 \%)$. Avec 52204 personnes arrivées pour demander l'asile conventionnel, auxquelles il convient d'ajouter, d'une part, 7564 mineurs accompagnants (soit un total de 59768 personnes) et, d'autre part, le nombre des demandes d'asile territorial (environ 25000 dossiers pour 2003 selon l'OFPRA), la France est devenue en 2003 le premier pays d'accueil en Europe devant le Royaume-Uni (61 050 nouvelles demandes, soit une diminution de $41 \%$ par rapport à 2002). Les deux chiffres (asile conventionnel et asile territorial) ne sont toutefois pas additionnables. L'ouverture d'une procédure n'exclue pas l'ouverture de l'autre; il y a donc des doubles comptes très difficiles à évaluer. 


\section{Point méthodologique sur les sources utilisées}

Les sources utilisées proviennent des rapports annuels de deux organismes, l'OFPRA, en charge de l'examen des demandes d'asile et la DPM (Direction de la Population et des Migrations), service placé sous l'autorité du Ministère de l'Emploi, du Travail et de la Cohésion Sociale, en charge de la politique d'accueil des demandeurs d'asile. Tous les ans, ces données sont pour partie synthétisées par Forum réfugiés dans son rapport annuel.

8 L'OFPRA fournit un tableau récapitulatif des demandes d'asile conventionnel, les demandes d'asile territorial étant centralisées par le Ministère de l'Intérieur. Pour l'année 2003, ce dernier n'a pas encore fourni de données statistiques et les seules sources disponibles sont des estimations ${ }^{5}$. Les données de l'OFPRA ne concernent que les premières demandes d'asile conventionnel, mais les procédures de réexamen sont très minoritaires, même si en forte augmentation (2 225 demandes de réexamen de dossier en 2003, correspondant à une augmentation de $25 \%$ par rapport à l'année 2002). Les données sont répertoriées par département selon l'adresse de résidence figurant sur le dossier du demandeur. Il s'agit de l'adresse de résidence effective au moment du dépôt de la demande. Elle ne correspond pas forcément à celle d'entrée dans la procédure au moment du dépôt de demande d'autorisation provisoire de séjour (APS) délivrée par la Préfecture du département de domiciliation (encadré 1). Les données fournies par l'OFPRA ne concernent que les adultes et les mineurs isolés. Les enfants mineurs accompagnant les demandeurs d'asile ne sont pas recensés ${ }^{6}$. Dans ses études, Forum réfugiés applique un cœfficient de 1,2 pour estimer le nombre total de demandeurs d'asile (deux enfants mineurs pour dix adultes), soit pour 52204 demandes d'asile déposées en 2003, un total de demandeurs d'asiles (adultes mineurs isolés et mineurs accompagnants) estimé à 62645 individus. Les données pour l'année 2003 ont été arrêtées au 20 mars 2004. 
Encadré 1 : L'entrée en procédure de demande d'asile (schéma simplifié - hors zone d'attente)

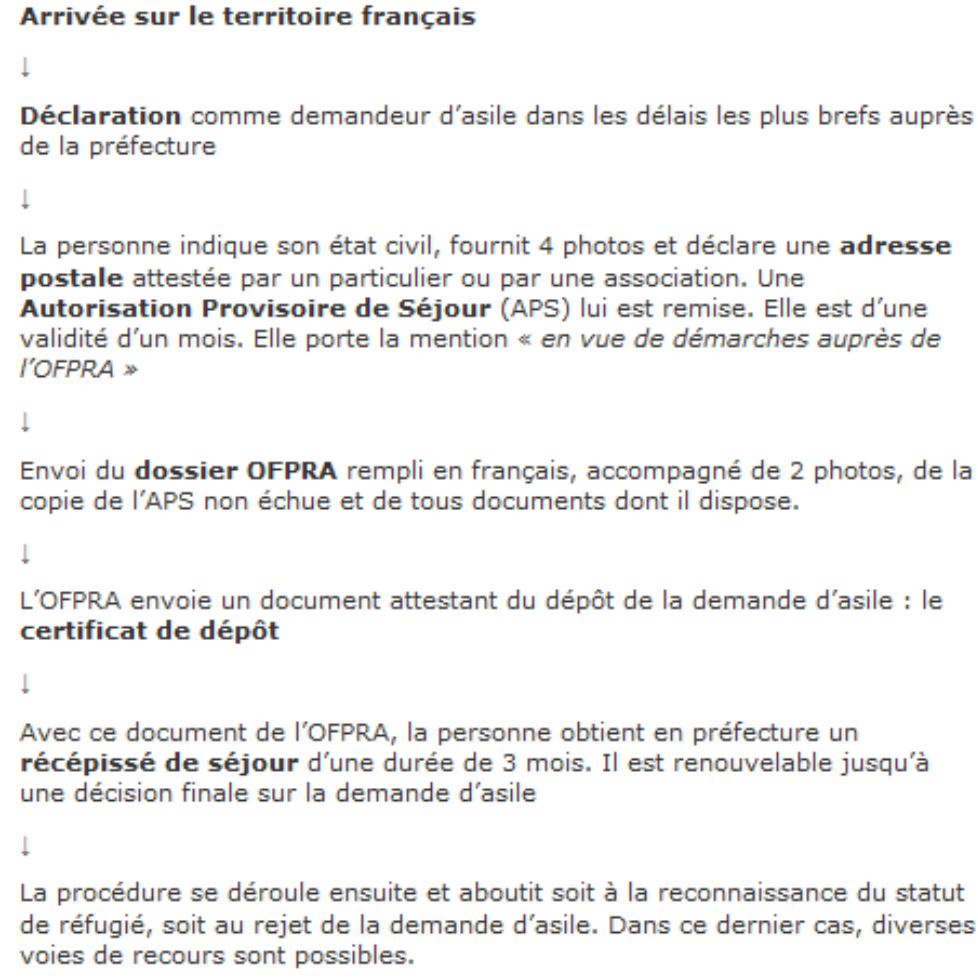

Certains dispositifs présentés ici seront modifiés dès la publication des décrets d'applications de la nouvelle loi adoptée en 2003.

Conception et réalisation : Xavier Richard, Forum Réfugiés.

9 La DPM a en charge l'accueil, l'hébergement et l'accès aux droits sociaux des demandeurs d'asile et des réfugiés statutaires. À ce titre, elle fournit annuellement des données statistiques sur les capacités d'hébergement existantes dans le cadre du dispositif national d'accueil (DNA) qui comprend les différentes structures d'hébergement spécialisées dans l'accueil des demandeurs d'asile et des réfugiés. Au $1^{\mathrm{er}}$ janvier 2004, le DNA comptait deux centres de transit (réservé aux primo-arrivants) d'une capacité de 186 places, 181 CADA (centre d'accueil pour demandeurs d'asile, réservé aux demandeurs d'asile au titre de la Convention de Genève) représentant 12 173 places, $28 \mathrm{CPH}$ (centre provisoire d'hébergement, réservé aux réfugiés statutaires) avec une capacité de 1043 places. À cette capacité du DNA, il convient d'ajouter 1200 places dans le dispositif AUDA (accueil d'urgence des demandeurs d'asile), géré par la Sonacotra.

Nous intéressant aux demandeurs d'asile, nous avons choisi de ne retenir dans la base de données que les types d'hébergement qui leur sont spécifiquement dédiés, à savoir centres de transit, centres d'accueil pour demandeurs d'asile (CADA), et places relevant du dispositif d'accueil d'urgence des demandeurs d'asile (AUDA) (encadré 2). Nous avons volontairement exclu les centres provisoires d'hébergement (CPH) qui ont pour mission l'accompagnement des réfugiés statutaires. Cependant, depuis quelques années, on trouve une proportion non négligeable de réfugiés statutaires dans le dispositif d'accueil pour demandeurs d'asile. Ainsi, pour l'année 2003, on compte une moyenne de $16 \%$ de réfugiés statutaires en CADA. Cette situation pose la question des conditions d'insertion des réfugiés statutaires et tout spécialement les conditions 
d'accès à un logement de droit commun, rendues de plus en plus difficiles en raison de la grave crise du logement social en France.

Encadré 2 : Les missions des structures spécialisées dans l'accueil des demandeurs d'asile

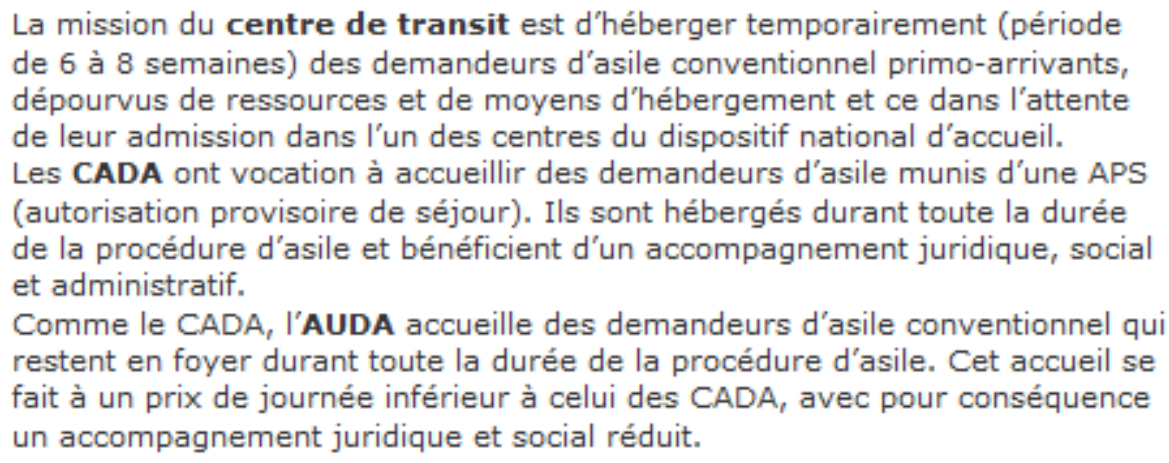

\section{Une géographie de la demande d'asile en France}

\section{Un phénomène très majoritairement urbain}

11 Si tous les départements français accueillent en 2003 des demandeurs d'asile (au sens de demandeurs ayant indiqué le département comme adresse de résidence sur le dossier de demande d'asile déposé à l'OFPRA), les écarts sont extrêmes entre les 7 demandes corses et les 13000 demandes domiciliées à Paris (écart-type : 1468).

12 La localisation de la demande d'asile, au vu des adresses figurant sur les dossiers OFPRA (que l'on appellera domiciliation pour simplifier), touche principalement Paris et l'Ilede-France (carte 1). Près d'un quart de la demande d'asile (24,9\%) est localisée dans Paris intra-muros et plus de la moitié de la demande d'asile déposée en 2003 est francilienne (27 977 demandes sur un total de 52 204). La capitale se place largement en tête, loin devant la Seine-Saint-Denis au $2^{\text {ème }}$ rang avec 5433 demandes. 


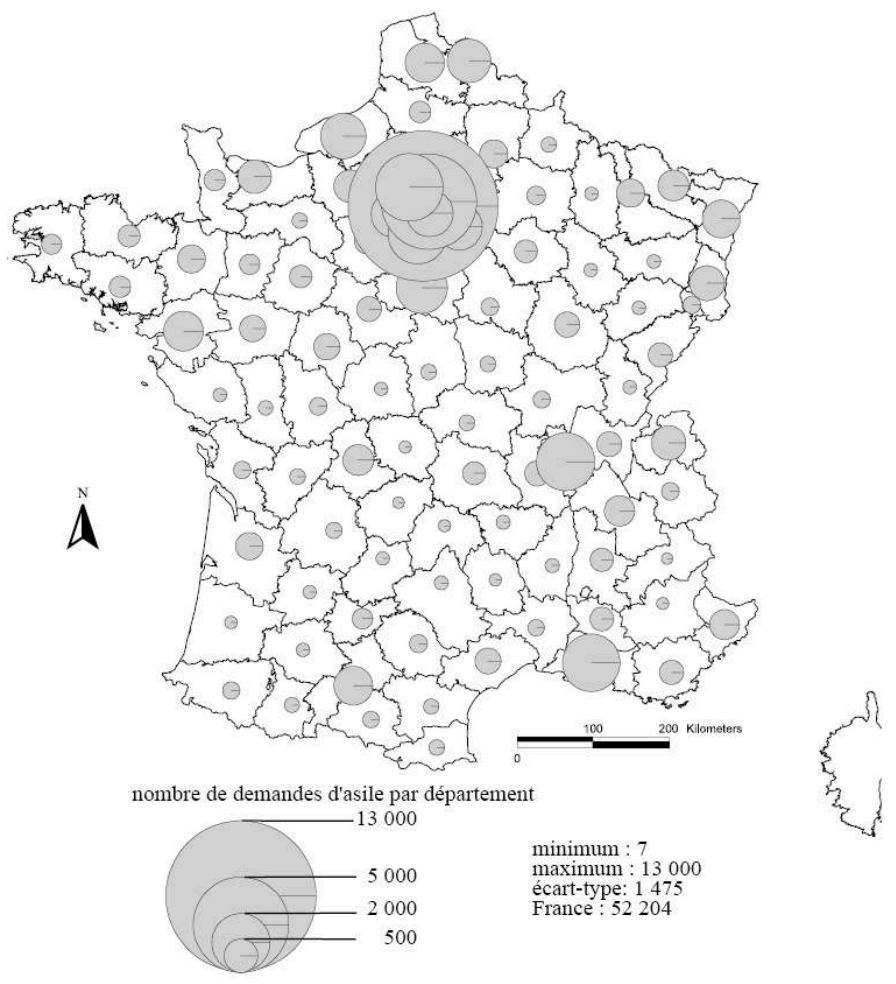

Conception-Réalisation : E. Bonerandi, L. Merchez, UMR 8503 - Géophile. Source. OFPRA.

On observe donc une extrême concentration de la demande d'asile sur une année donnée. En élargissant la base d'observation, on constate que 22 départements concentrent à eux-seuls $80 \%$ de la demande d'asile déposée en 2003 (tableau 1). Outre les départements franciliens, se retrouvent dans ce groupe de tête les départements des principales agglomérations françaises: Rhône (Lyon), Bouches-du-Rhône (Marseille), Nord (Lille), Loire-Atlantique (Nantes), Haute-Garonne (Toulouse) et Bas-Rhin (Strasbourg). Outre la localisation urbaine préférentielle qui s'explique par les logiques migratoires et par le recours aux services locaux d'appui à l'asile, on note également une préférence spatiale pour les départements frontaliers, que la frontière soit terrestre (Lille, Toulouse et Strasbourg) ou maritime (Marseille et Nantes). Si les critères d'agglomération urbaine et de situation frontalière se recoupent largement, le critère frontalier joue également à lui seul, comme dans le Haut-Rhin et la HauteSavoie, départements nettement moins urbanisés. On note également un élargissement de l'aire francilienne sur les départements limitrophes, à savoir essentiellement le Loiret, la Seine-Maritime et l'Oise. 
Tableau 1 : Le poids relatif et absolu de la demande d'asile par département en 2003 (Seuls ont été représentés les 22 premiers et les 22 derniers départements)

\begin{tabular}{|c|c|c|c|c|c|c|c|}
\hline rang & département & $\%$ & nombre & rang & département & $\%$ & nomt \\
\hline 1 & Paris & 24,90 & 13000 & 74 & Deux-Sèvres & 0,09 & 46 \\
\hline 2 & $\begin{array}{l}\text { Seine-Saint- } \\
\text { Denis }\end{array}$ & 10,41 & 5433 & 75 & Ardèche & 0,08 & 40 \\
\hline 3 & Val-d'Oise & 5,18 & 2706 & 76 & Haute-Loire & 0,07 & 39 \\
\hline 4 & Essonne & 3,88 & 2024 & 77 & Aveyron & 0,07 & 38 \\
\hline 5 & Rhône & 3,77 & 1969 & 78 & Vendée & 0,07 & 35 \\
\hline 6 & $\begin{array}{l}\text { Bouches-du- } \\
\text { Rhône }\end{array}$ & 3,69 & 1928 & 79 & Jura & 0,06 & 33 \\
\hline 7 & Loiret & 2,88 & 1504 & 80 & Vosges & 0,06 & 33 \\
\hline 8 & Val-de-Marne & 2,33 & 1217 & 81 & Haute-Saône & 0,06 & 32 \\
\hline 9 & $\begin{array}{l}\text { Hauts-de- } \\
\text { Seine }\end{array}$ & 2,31 & 1204 & 82 & Haute-Marne & 0,06 & 31 \\
\hline 10 & Yvelines & 2,29 & 1198 & 83 & Meuse & 0,06 & 31 \\
\hline 11 & $\begin{array}{l}\text { Seine-et- } \\
\text { Marne }\end{array}$ & 2,29 & 1195 & 84 & Gers & 0,06 & 29 \\
\hline 12 & $\begin{array}{l}\text { Seine- } \\
\text { Maritime }\end{array}$ & 2,20 & 1148 & 85 & Indre & 0,06 & 29 \\
\hline 13 & Nord & 1,99 & 1039 & 86 & Lot & 0,06 & 29 \\
\hline 14 & $\begin{array}{l}\text { Loire- } \\
\text { Atlantique }\end{array}$ & 1,61 & 842 & 87 & $\begin{array}{l}\text { Lot-et- } \\
\text { Garonne }\end{array}$ & 0,05 & 28 \\
\hline 15 & Pas-de-Calais & 1,56 & 813 & 88 & Landes & 0,04 & 19 \\
\hline 16 & Oise & 1,52 & 795 & 89 & Creuse & 0,03 & 17 \\
\hline 17 & $\begin{array}{l}\text { Haute- } \\
\text { Garonne }\end{array}$ & 1,52 & 793 & 90 & Cantal & 0,03 & 16 \\
\hline 18 & Bas-Rhin & 1,42 & 742 & 91 & Lozère & 0,03 & 16 \\
\hline 19 & Haut-Rhin & 1,18 & 614 & 92 & $\begin{array}{l}\text { Alpes de } \\
\text { Haute- } \\
\text { Provence }\end{array}$ & 0,03 & 14 \\
\hline 20 & Haute-Savoie & 1,12 & 587 & 93 & Corrèze & 0,02 & 10 \\
\hline 21 & Dom-Tom & 1,07 & 558 & 94 & Hautes-Alpes & 0,02 & 8 \\
\hline \multirow[t]{2}{*}{22} & Calvados & 1,06 & 551 & 95 & Corse & 0,01 & 7 \\
\hline & TOTA & 100, & 52 & 20 & & & \\
\hline
\end{tabular}

Source : OFPRA, 2003

14 À l'opposé, les départements qui connaissent les plus faibles nombres de domiciliations de demandeurs d'asile sont tous des départements majoritairement ruraux, que l'on associe volontiers à la France du vide ou des faibles densités.

On peut tenter de relativiser la demande d'asile par département en comparant son poids au regard de la population départementale enregistrée lors du recensement général de la population de 1999 (carte 2). Si le rapport entre demandeurs d'asile et population totale est de 0,87 pour mille au niveau national, les écarts restent significatifs, même s'ils sont moindres que dans une présentation absolue. La valeur maximale est atteinte par le département-capitale avec 6,12 pour mille et la valeur minimale est de 0,03 pour les deux départements corses réunis. L'écart-type est de 0,8, ce qui demeure élevé. Le commentaire varie peu. Paris constitue une classe à lui seul, suivi par les départements limitrophes, tels Seine-Saint-Denis $(3,93 \%$ ), Val-d'Oise $(2,45)$ et l'Essonne $(1,78)$, mais aussi le Loiret $(2,43)$. Cependant, l'unité francilienne se fragmente. La charge de la demande d'asile rapportée à la population résidente est bien moindre dans la Seine-et-Marne $(1,00)$, le Val-de-Marne $(0,99)$, les Yvelines $(0,88)$ et les Hauts-de-Seine $(0,84)$. Si la charge de la demande d'asile recoupe les départements d'effectifs élevés de demandes d'asile, la carte fait apparaître une aire de forte charge sur le bassin parisien élargi dans une dissymétrie «profitant» à l'ouest vers la SeineMaritime et le sud-ouest dans la direction de la vallée de la Loire. On observe le même phénomène d'ampleur réduite autour de la Haute-Garonne $(0,76)$, incluant les départements du Tarn-et-Garonne $(0,62)$ et de l'Ariège $(0,59)$. 


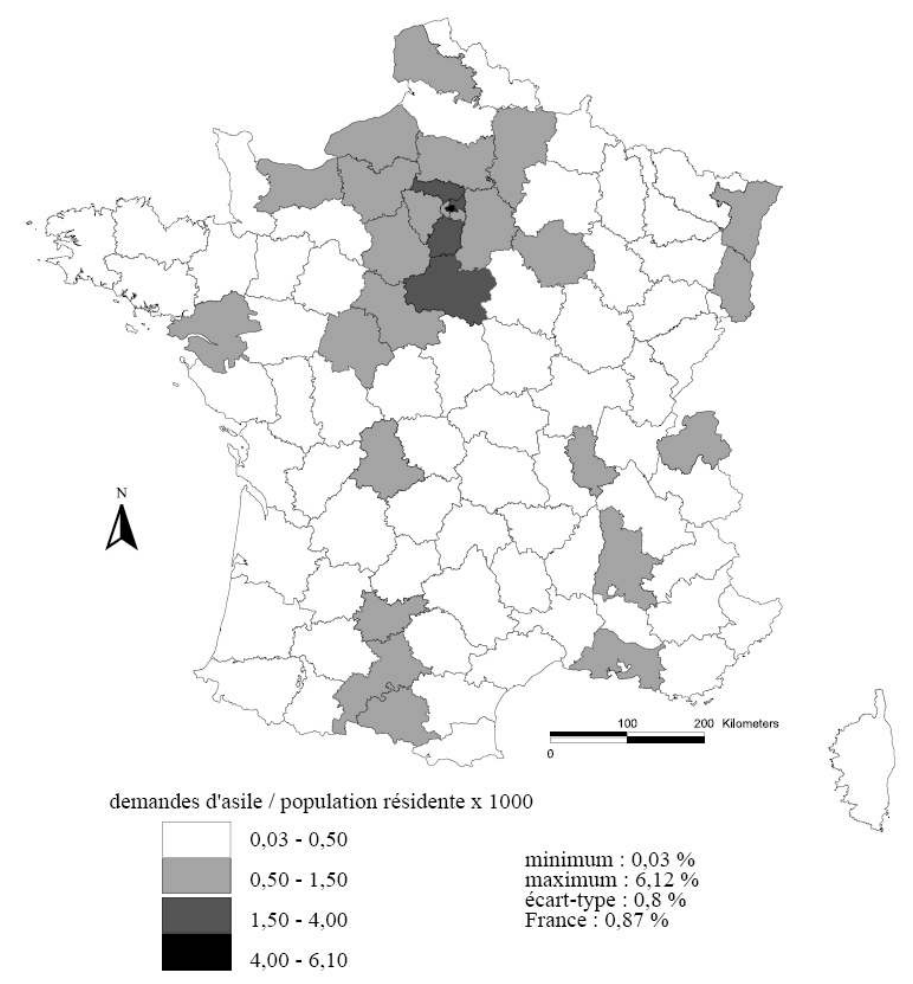

Conception-Réalisation : E. Bonerandi, L. Merchez, UMR 8503 - Géophile. Source. OFPRA et INSEE, RGP 1999.

\section{L'évolution de la demande d'asile, l'importance de la période de référence}

L'évolution de la demande d'asile sur les vingt dernières années est loin d'être linéaire (graphique 1). On note une augmentation des demandes d'asile à partir du milieu des années 1980, avec un pic de la demande en 1989 avec plus de 60000 requêtes (Legoux, 1993), que certains justifient en partie par un report des immigrés économiques sur la demande d'asile (cas des Maliens et des Turcs en particulier), puis une diminution rapide durant la première moitié de la décennie 1990 (une moyenne de 20000 demande par an) expliquée par plusieurs phénomènes. Tout d'abord, la France a mis en place des mesures limitant l'accès au territoire (amendes aux transporteurs, restriction des visas) et a restreint les possibilités d'aides matérielles (suppression des aides au logement et des prestations familiales), de formation (suppression de l'accès à la formation rémunérée) et d'emploi (restriction de l'accès au marché du travail en 1991). Ensuite, la modernisation de l'OFPRA en 1990 s'est traduite par un accroissement des moyens humains et matériels qui a entraîné un traitement plus rapide des dossiers et un apurement massif des dossiers en attente. Enfin, et comme le rappelle O. Brachet, « Fin 1989, la chute du mur de Berlin crée un véritable séisme dans les esprits. Les craintes des pouvoirs publics de voir à cette occasion se développer des arrivées massives en provenance de l'Est, leur suggèrent de prendre des mesures encore plus dissuasives" (Brachet, 2002). Cependant, la politique restrictive mise en place dans les années 1989/90 n'a pas apporté les preuves de sa réussite en matière de dissuasion puisqu'à partir du milieu 
des années 1990 on observe une inversion de tendance: le nombre des dossiers de demande d'asile conventionnel passe de 17745 en 1996 à 52204 en 2003.

Graphique 1 : Évolution des premières demandes d'asile conventionnel, 1981/2003

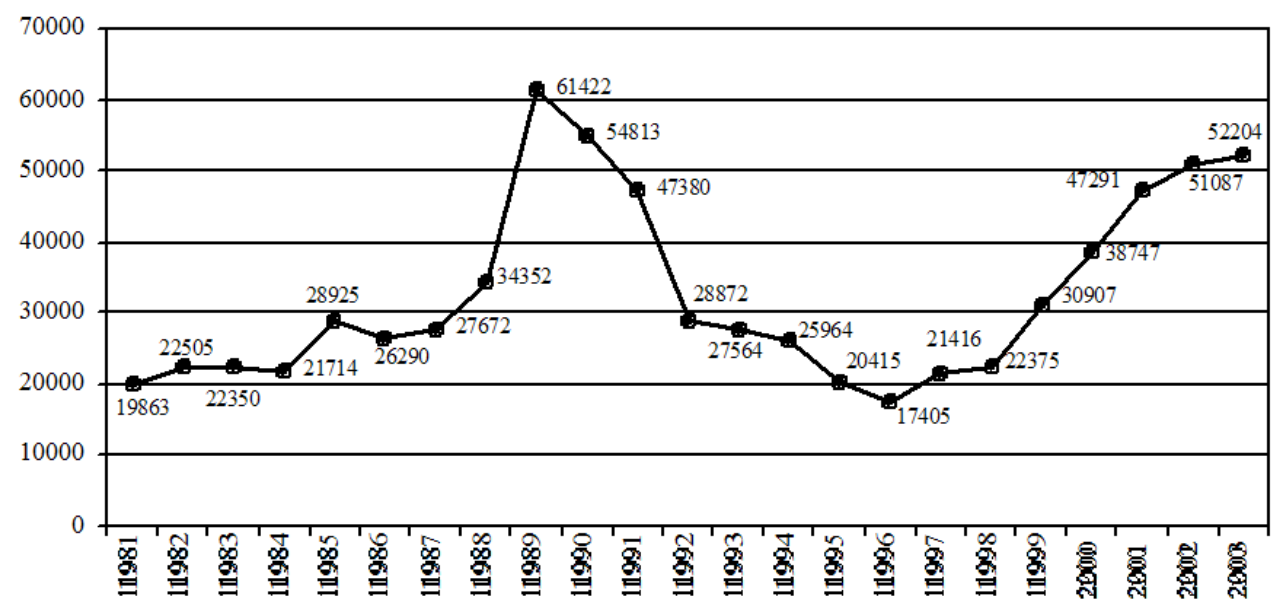

NB : Il s'agit uniquement des nouvelles demandes d'asile conventionnel d'adultes et de mineurs isolés. Source : OFPRA, rapport d'activité 2003.

Nous avons choisi une période de dix ans pour exprimer la temporalité moyenne en partant des données disponibles en 2003 et en remontant à 1993. Ce choix, s'il présente l'intérêt d'une décennie, est cependant discutable car l'année 1993 se situe dans le mouvement de décroissance du pic enregistré en 1989 qui se poursuit jusqu'en 1996. Les taux d'évolution sont donc minorés en comparaison d'une observation qui aurait porté sur la période 1996-2003. Nous avons également choisi de ne représenter que le solde absolu d'évolution, tant les variations relatives dépendent des données absolues de départ et d'arrivée. Par exemple, le département de la Manche enregistre 146 demandes d'asile en 2003, contre 4 en 1993, ce qui correspond à une augmentation de 3 $550 \%$. En comparaison, le solde pour Paris intra-muros s'il est de 2581 ne correspond qu'à une augmentation de $24,8 \%$. Pour plus de rigueur, il conviendrait de suivre les trajectoires inter-annuelles nationale et départementales en les comparant au moyen d'une classification ascendante hiérarchique.

Si onze départements enregistrent une diminution du nombre annuel de demandeurs d'asile entre l'année 1993 et l'année 2003, avec le cas particulier de la Gironde qui passe de 706 demandes en 1993 à 324 en 2003, l'essentiel des départements français connaît une augmentation du nombre de demandeurs d'asile entre les deux années de référence. La répartition spatiale de l'évolution de la demande d'asile entre 1993 et 2003 (carte 3) montre là encore la suprématie francilienne qui touche préférentiellement la Seine-Saint-Denis ( $3023,+125 \%)$, Paris $(2581,+24,8 \%)$ et l'Essonne ( 1711 , avec une augmentation relative très forte puisqu'elle atteint $546 \%$ ). On retrouve les départements accueillant l'essentiel des demandeurs d'asile en 2003 dans les fortes croissances 1993-2003, attestant ainsi d'un processus cumulatif (la demande appelle la demande), on note également une recomposition à la marge qui se joue selon deux logiques spatiales: soit ponctuellement sur des départements isolés, comme la Loire-Atlantique, soit dans le sens d'un élargissement de l'aire d'accueil francilienne sur des départements limitrophes comme le Loiret ou la Seine-Maritime. 
Rapportée à la population départementale en 1999, ces élargissements régionaux se lisent avec encore plus de force (carte 4).

Carte 3 : Évolution de la demande d'asile, 1993-2003

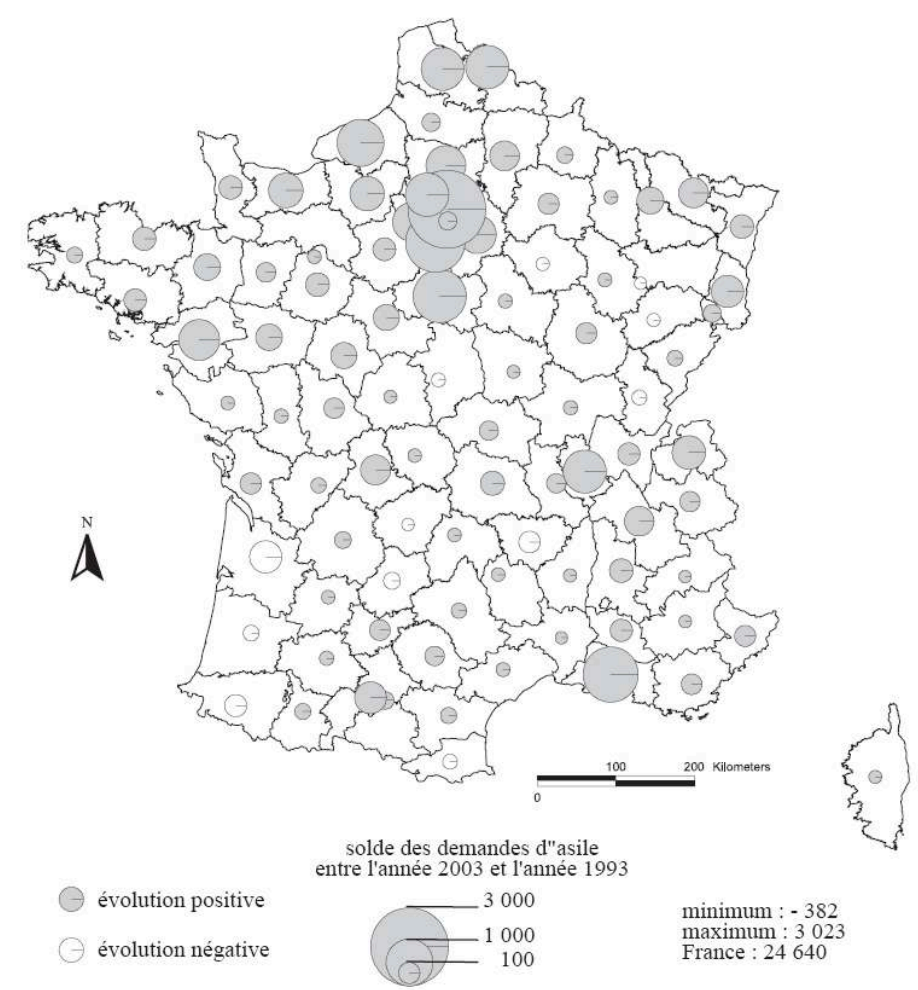

Source : OFPRA

Conception-Réalisation : E. Bonerandi, L. Merchez, UMR 8503 - Géophile. 
Carte 4 : L'évolution de la demande d'asile (1993-2003) rapportée à la population résidente (RGP 1999)

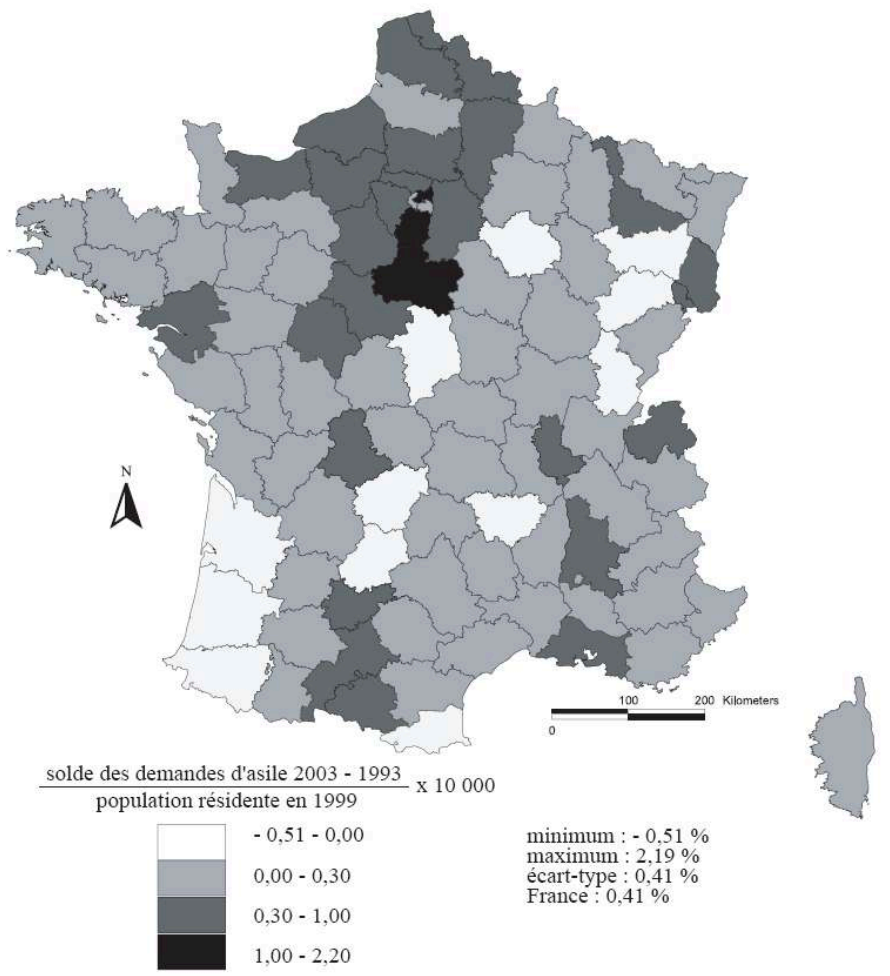

Source : OFPRA et INSEE, RGP 1999

Conception-Réalisation : E. Bonerandi, L. Merchez, UMR 8503 - Géophile.

19 La hiérarchie de la demande d'asile se modifie sensiblement (tableau 2). L'apparition des deux départements nordistes dans les quinze premiers départements français pour l'enregistrement de la demande d'asile peut s'expliquer de façon conjoncturelle par les conséquences de la fermeture du centre de Sangatte. 
Tableau 2 : Demande d'asile : une recomposition à la marge Les quinze premiers départements d'enregistrement de la demande d'asile en 1993 et en 2003

\begin{tabular}{|c|c|c|c|c|c|}
\hline & 1993 & & & 2003 & \\
\hline rang & département & demandes & rang & département & demandes \\
\hline 1 & Paris & 10419 & 1 & Paris & 13000 \\
\hline 2 & $\begin{array}{l}\text { Seine-Saint- } \\
\text { Denis }\end{array}$ & 2410 & 2 & $\begin{array}{l}\text { Seine-Saint- } \\
\text { Denis }\end{array}$ & 5433 \\
\hline 3 & Val-d'Oise & 1881 & 3 & Val-d'Oise & 2706 \\
\hline 4 & Val-de-Marne & 1171 & 4 & Val-de-Marne & 2024 \\
\hline 5 & Rhône & 1161 & 5 & Rhône & 1969 \\
\hline 6 & Hauts-de-Seine & 817 & 6 & Hauts-de-Seine & 1928 \\
\hline 7 & Gironde & 706 & 7 & Gironde & 1504 \\
\hline 8 & Seine-et-Marne & 604 & 8 & Seine-et-Marne & 1217 \\
\hline 9 & Bas-Rhin & 596 & 9 & Bas-Rhin & 1204 \\
\hline 10 & Yvelines & 561 & 10 & Yvelines & 1198 \\
\hline 11 & $\begin{array}{l}\text { Bouches-du- } \\
\text { Rhône }\end{array}$ & 481 & 11 & $\begin{array}{l}\text { Bouches-du- } \\
\text { Rhône }\end{array}$ & 1195 \\
\hline 12 & Haute-Garonne & 422 & 12 & Haute-Garonne & 1148 \\
\hline 13 & Essonne & 313 & 13 & Essonne & 1039 \\
\hline 14 & Alpes-Maritimes & 311 & 14 & Alpes-Maritimes & 842 \\
\hline \multirow[t]{3}{*}{15} & Hérault & 253 & 15 & Hérault & 813 \\
\hline & $\begin{array}{l}\text { Autres } \\
\text { départements }\end{array}$ & 3465 & & $\begin{array}{l}\text { Autres } \\
\text { départements }\end{array}$ & 12981 \\
\hline & $\begin{array}{l}\text { France } \\
\text { entière }\end{array}$ & 27564 & & $\begin{array}{l}\text { France } \\
\text { entière }\end{array}$ & 52204 \\
\hline
\end{tabular}

L'évolution de la demande d'asile connaît d'importantes fluctuations inter-annuelles. On peut considérer qu'elles recouvrent une part d'aléatoire car beaucoup de variables entrent en jeu, à commencer par l'environnement international (apparition de nouveaux conflits, apaisement ailleurs), l'évolution de la législation européenne (fermeture des frontières annoncée par l'Union Européenne qui entrâne sur l'année 2003 une baisse de $22 \%$ du nombre de demandes d'asile dans l'Europe des 15, et une baisse de $19 \%$ dans l'Europe des 25 ) et nationale (comme la fin de l'asile territorial effective au $1^{\mathrm{er}}$ janvier 2004). Ainsi il faut sans cesse recouper les données qui, si elles sont présentées séparément, peuvent conduire à des commentaires et à des conclusions bien différentes. Dans un contexte de ralentissement de la demande d'asile conventionnel au niveau européen, la France enregistre ainsi une poursuite de la tendance à l'augmentation pour l'année 2003 (+1,3\%). Au niveau départemental, ce ne sont plus 11 départements qui enregistrent un solde négatif mais 45 sur un total de 95 départements métropolitains (carte 5). Si Paris continue à enregistrer une augmentation de la demande, des départements comme le Val-de-Marne, la Seine-etMarne, les Bouches-du-Rhône ou le Rhône connaissent pour l'année 2003 un solde négatif par rapport à 2002, alors que la Seine-Saint-Denis est stable et que le Loiret, le Nord, le Pas-de-Calais et les Alpes-Maritimes enregistrent des hausses importantes. Cependant, les conclusions tirées des données prises sur une année peuvent dès l'année suivante être remises en question. Ainsi, si le département du Rhône enregistre une baisse de 10,5\% de la demande (-231) en 2003, les cinq premiers mois de l'année 2004 montrent au contraire un retournement de tendance avec une reprise de l'augmentation. 


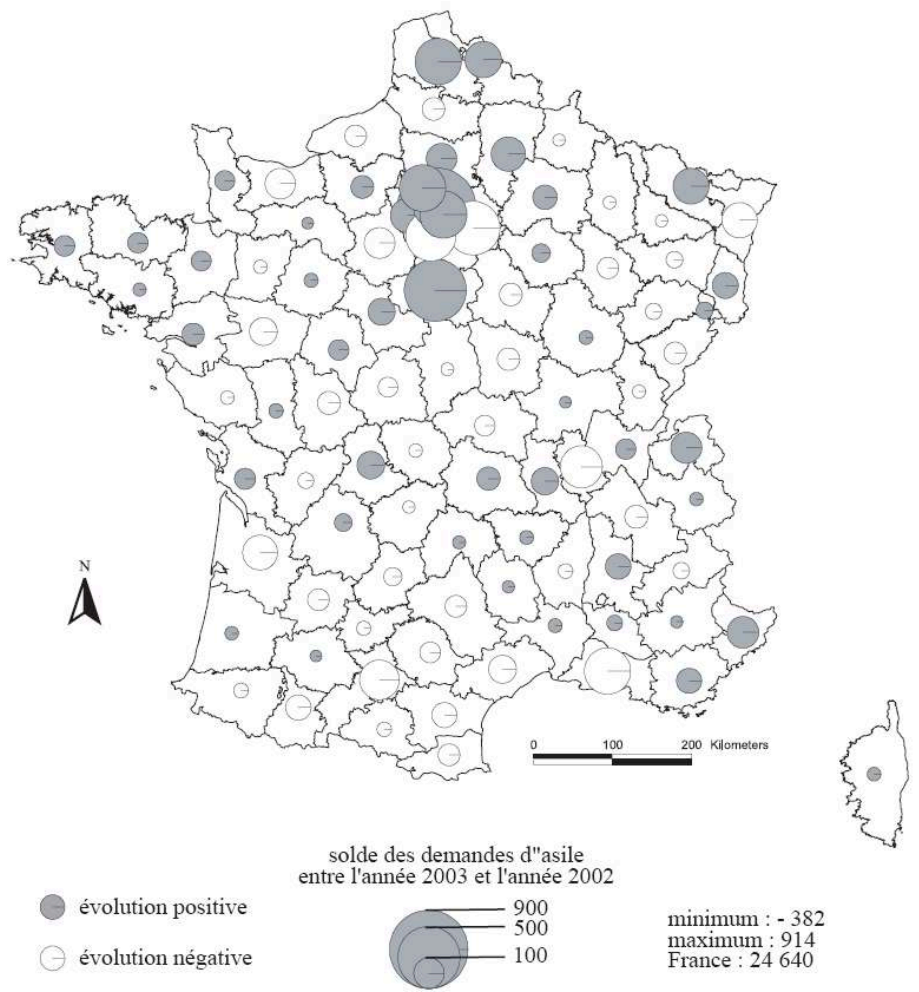

Source : OFPRA

Conception-Réalisation : E. Bonerandi, L. Merchez, UMR 8503 - Géophile.

\section{Entre tensions et rééquilibrage : demande d'asile et hébergement}

L'intérêt du croisement entre les dépôts annuels de demandes d'asile et les capacités en hébergement spécifiques est l'étude de la corrélation entre deux données. Il s'agit de considérer si l'offre d'hébergement dédiée aux demandeurs d'asile correspond à la demande d'asile, par exemple si pour un département enregistrant de fortes demandes répond des capacités d'hébergement proportionnellement adaptées, et inversement. Trois situation sont théoriquement possibles: l'adaptation entre demande et hébergement (capacités d'hébergement couvrant la demande), la tension (capacités d'hébergement inférieures à la demande) ou la marge de manœuvre (capacités d'hébergement supérieures à la demande).

Il convient de préciser d'entrée de jeu un point méthodologique très important. En effet, il ne s'agit ici que d'un rapport entre deux données et non de la représentation d'une réalité car l'on travaille d'une part sur un flux (la demande d'asile) et d'autre part sur un stock (les capacités d'hébergement).

\section{Le manque récurrent de structures d'hébergement}

Malgré l'augmentation régulière des capacités d'hébergement ces dernières années (l'offre d'hébergement en CADA a été multiplié par trois depuis 1998 et, en 2003, 2000 
places nouvelles ont été créées), le contexte de l'accueil reste très tendu. Les capacités sont en nombre insuffisant par rapport aux nouvelles arrivées puisque environ $20 \%$ seulement des besoins théoriques sont couverts (graphique 2).

Graphique 2 : Des capacités d'hébergement largement déficitaires
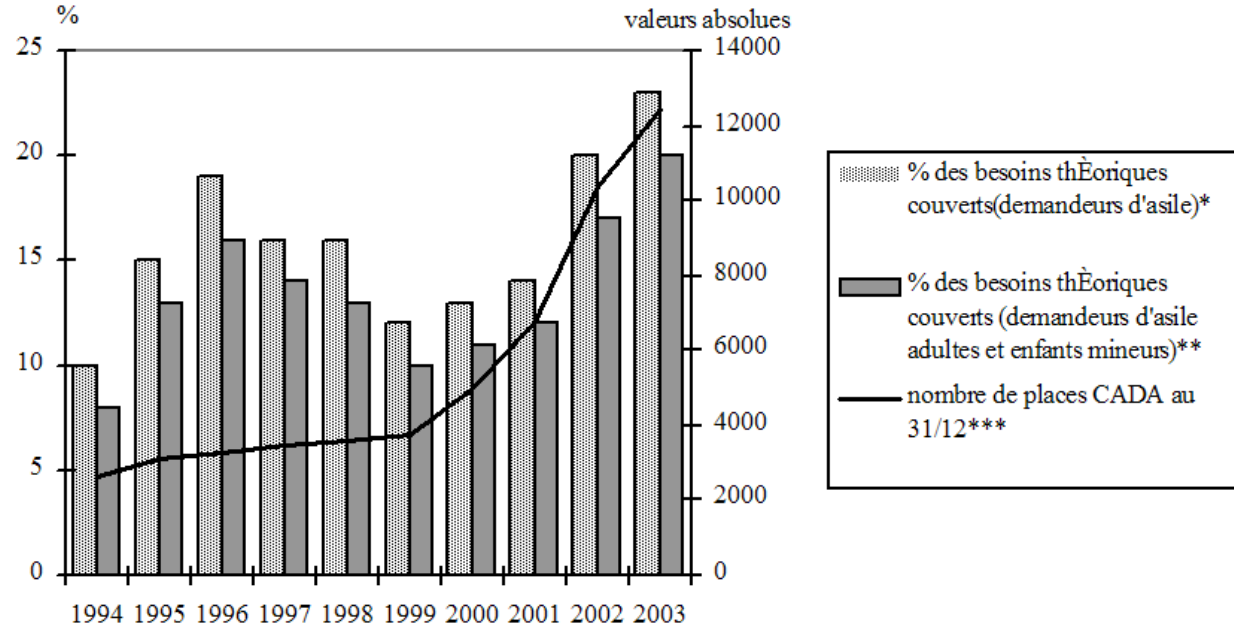

* Source OFPRA (les chiffres publiés par I'OFPRA concernent uniquement les adultes et mineurs isolés).

** Hypothèse à partir du service domiciliation de Forum réfugiés (cœfficient de 1,2 par rapport aux chiffres OFPRA, soit deux enfants mineurs pour dix adultes).

*** Source DPM

Afin de respecter l'obligation induite par la loi d'orientation relative à la lutte contre les exclusions de 1998, qui enjoint les autorités à veiller à ce qu'aucune famille ne soit sans abri, les pouvoirs publics, et en premier lieu les DDASS, se trouvent dans l'obligation d'ouvrir ou de recourir à des dispositifs spécifiques. La plupart du temps, ceux-ci sont élaborés dans l'urgence, ne sont pas adaptés à la prise en charge des demandeurs d'asile et sont coûteux. On assiste ainsi à la multiplication des solutions alternatives (comme le placement en structures d'urgence, ou en hôtels...) relevant du bricolage institutionnel, pour tous ceux qui ne peuvent se loger par leurs propres moyens et qui ne peuvent accéder au DNA ${ }^{7}$. Selon les sources du Ministère de l'Emploi, du Travail et de la Cohésion Sociale, environ 15000 personnes seraient ainsi hébergées à l'hôtel et 4000 dans les CHRS (Centres d'Hébergement et de Réinsertion sociale, structures relevant de l'urgence sociale). Il y aurait par conséquent autant de demandeurs d'asile hébergés à l'hôtel qu'en CADA.

\section{La géographie de l'hébergement, une France inverse?}

La répartition départementale des structures d'hébergement spécifiques (CADA, AUDA et transit) présente une France tout à fait différente de celles de la demande d'asile (carte 6). Les écarts inter-départementaux sont beaucoup moins élevés : l'écart-type pour la demande d'asile en 2003 est de 1 475, alors qu'il n'est que de 119 pour les structures d'hébergement. La logique qui prévaut ici est celle d'une couverture de la totalité des départements français, à l'exception de la Corse. À l'inverse des cartes précédentes, l'Ile-de-France perd de sa suprématie. Même si les capacités les plus importantes se trouvent encore dans les départements majoritairement urbains, la 
hiérarchie est très sensiblement différente. Ainsi, le Rhône arrive en tête avec 721 places, suivi par la Seine-Maritime (485 places). Parmi les dix premiers départements, on ne trouve que trois départements franciliens: la Seine-et-Marne ( $3^{\text {ème }}$ rang, 440 places), le Val-d'Oise ( $8^{\text {ème }}$ rang, 307 places) et le Val-de-Marne (9 ${ }^{\text {ème }}$ rang, 305 places). Paris et la Seine-Saint-Denis font piètre figure en ne figurant qu'au $12^{\text {ème }}$ (270 places) et $27^{\text {ème }}$ rang (206 places). À l'inverse, des départements qualifiés de ruraux se trouvent mieux placés, c'est le cas emblématique de l'Aube ( $13^{\text {ème }}$ rang, 255 places, juste derrière Paris).

\section{Carte 6 : La capacité d'hébergement en 2003}

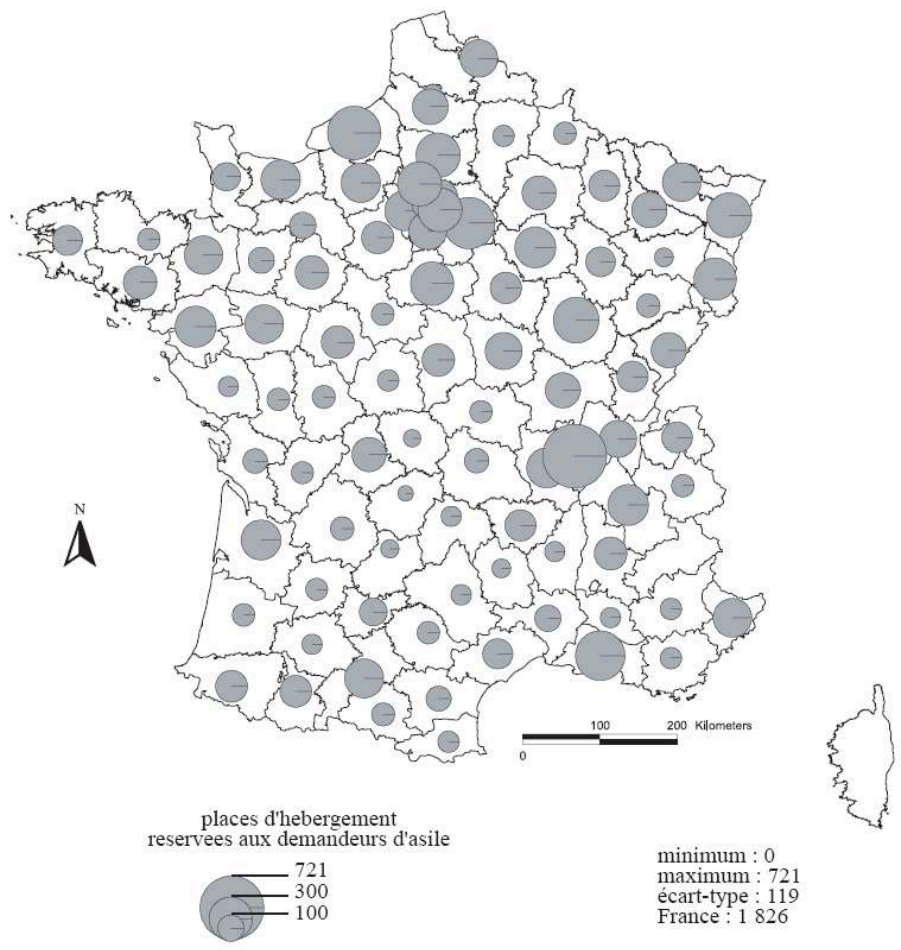

Source : OFPRA.

Conception-Réalisation : E. Bonerandi, L. Merchez, UMR 8503 - Géophile.

Si l'on compare les poids relatifs des départements en capacités d'hébergement et en demandes d'asile pour l'année 2003 rapportés au total de la France, on constate de profondes disparités qui font ressortir le sous-équipement en structures d'hébergement des départements enregistrant les plus forts taux de demandes (tableau 3). Ainsi, si Paris intra-muros enregistre $24,9 \%$ des demandes d'asile de 2003 , la capitale ne possède que $1,82 \%$ des capacités d'hébergement nationales, le constat est le même pour la Seine-Saint-Denis. À l'inverse, plusieurs départements représentent un poids en hébergement supérieur à celui de la demande d'asile. Si l'on trouve dans cette catégorie le département du Rhône que l'on peut considérer comme relativement bien équipé ou, dans une moindre mesure, ceux des Bouches-du-Rhône et du Loiret, les écarts les plus importants concernent les départements enregistrant peu de demandes d'asile, comme la Côte d'Or, l'Ille-et-Vilaine, l'Aube, l'Ain ou les Alpes-Maritimes, pour n'en citer que quelques-uns. 
Tableau 3 : Les poids relatifs de la demande d'asile et de l'hébergement par département

\begin{tabular}{|c|c|c|c|}
\hline rang & département & $\begin{array}{l}\text { \% hébergement } \\
2003\end{array}$ & $\begin{array}{l}\text { \% demandes } \\
2003\end{array}$ \\
\hline 1 & Rhône & 5,42 & 3,77 \\
\hline 2 & Seine-Maritime & 3,26 & 2,20 \\
\hline 3 & Seine-et-Marne & 3,13 & 2,29 \\
\hline 4 & Bouches-du-Rhône & 3,00 & 3,69 \\
\hline 5 & Bas-Rhin & 2,66 & 1,42 \\
\hline 6 & Côte-d'or & 2,50 & 0,48 \\
\hline 7 & Val-de-Marne & 2,39 & 2,33 \\
\hline 8 & Oise & 2,30 & 1,52 \\
\hline 9 & Loiret & 2,25 & 2,88 \\
\hline 10 & Val-d'Oise & 2,06 & 5,18 \\
\hline 11 & Haut-Rhin & 2,00 & 1,18 \\
\hline 12 & Loire-Atlantique & 1,98 & 1,61 \\
\hline 13 & Ille-et-Vilaine & 1,85 & 0,65 \\
\hline 14 & Paris & 1,82 & 24,90 \\
\hline 15 & Haute-Garonne & 1,78 & 1,52 \\
\hline 16 & Gironde & 1,74 & 0,62 \\
\hline 17 & Aube & 1,71 & 0,36 \\
\hline 18 & Isère & 1,69 & 0,82 \\
\hline 19 & Nord & 1,66 & 1,99 \\
\hline 20 & Ain & 1,65 & 0,46 \\
\hline 21 & Loire & 1,61 & 0,47 \\
\hline 22 & Yvelines & 1,61 & 2,29 \\
\hline 23 & Alpes-Maritimes & 1,59 & 0,78 \\
\hline 24 & Calvados & 1,56 & 1,06 \\
\hline 25 & Eure & 1,55 & 0,97 \\
\hline 26 & Maine-et-Loire & 1,48 & 0,55 \\
\hline 27 & Moselle & 1,48 & 0,85 \\
\hline 28 & Nièvre & 1,43 & 0,13 \\
\hline 29 & Somme & 1,41 & 0,29 \\
\hline 30 & Essonne & 1,41 & 3,88 \\
\hline 31 & Doubs & 1,39 & 0,42 \\
\hline 32 & $\begin{array}{l}\text { Seine-Saint- } \\
\text { Denis }\end{array}$ & 1,39 & 10,41 \\
\hline & TOTAL & 100,00 & 100,00 \\
\hline
\end{tabular}

Source : OFPRA et DPM.

27 Pour compléter cette présentation, nous avons réalisé la carte du taux de couverture en hébergement en 2003 afin de faire apparaître les situations départementales théoriquement excédentaires, équilibrées ou déficitaires (carte 7). Là-aussi, le résultat diffère largement des cartes consacrées à la demande d'asile. Le taux de couverture est largement déficitaire dans les départements qui enregistrent le plus de demandes, alors que les situations largement excédentaires (en termes d'hébergement théorique) sont toutes localisées dans la France des faibles densités. Les taux de couverture dépassant $200 \%$ concernent ainsi les départements suivants, par ordre décroissant : Meuse, Jura, Alpes de Haute-Provence, Haute-Marne, Landes, Haute-Loire, Nièvre, Cantal, HautesPyrénées, Haute-Saône et Cher. 


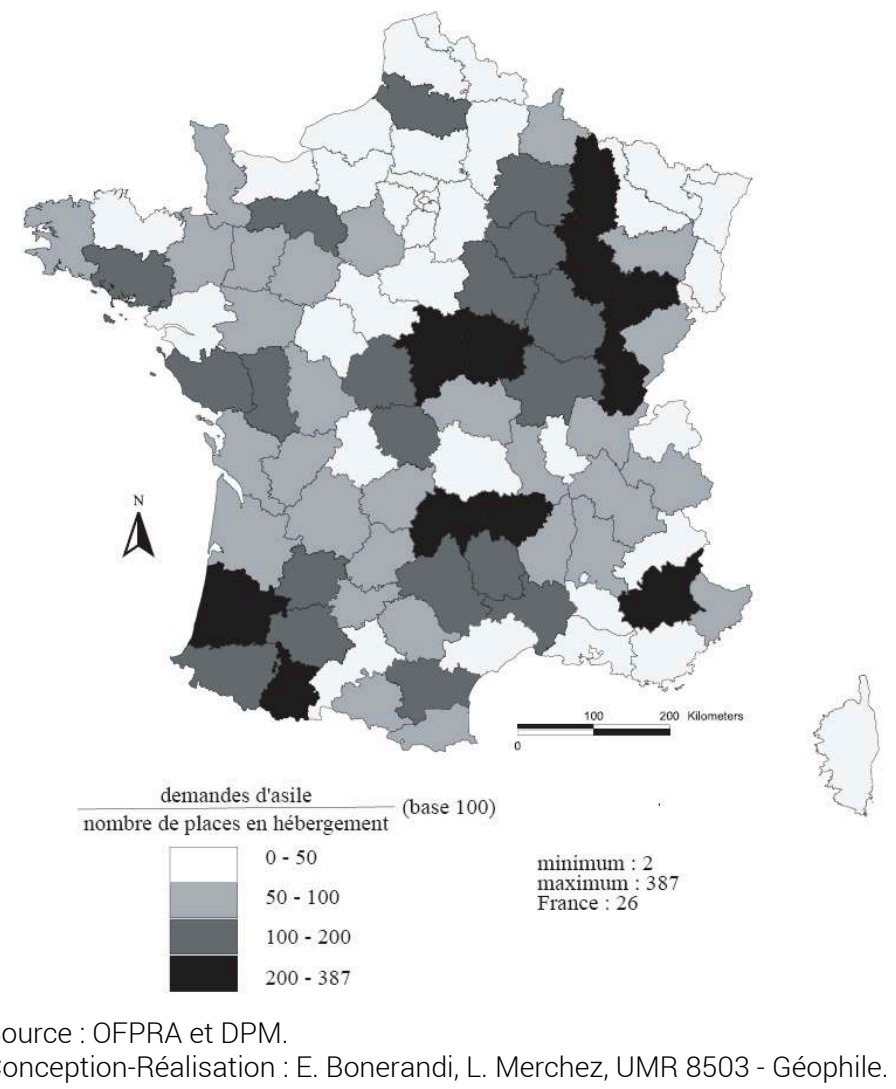

\section{Vers un rééquilibrage venu du haut?}

Dans un contexte national de création importante de places en CADA depuis 1998, nous nous sommes plus particulièrement intéressés à celles créées ou décidées par anticipation pour l'année 2003 dans le cadre du DNA. Ces nouvelles places ont-elles permis un rééquilibrage local (réduire la tension par une augmentation de la capacités en CADA dans le même département), une redistribution régionale (dans un département limitrophe) voire nationale (sur des départements disjoints) ?

L'extension des places CADA s'est opérée dans un souci de répartition sur l'ensemble du territoire national, et aujourd'hui seuls quatre départements métropolitains n'en sont pas équipés (Hautes-Alpes, Corse du Sud, Haute-Corse, Territoire de Belfort) auxquels s'ajoutent les trois départements d'outre-mer. La répartition des nouvelles places ne répond que très marginalement aux situations de tensions locales. Ainsi, aucune place n'a été créée dans Paris intra-muros. Si l'on peut reconnaître la difficulté à trouver une offre foncière disponible dans la capitale pour construire un centre d'hébergement dédié aux demandeurs d'asile, il n'en demeure pas moins que la tension reste extrêmement vive. Et ce ne sont pas les 60 nouvelles places créées en Seine-Saint-Denis qui résorberont un taux de couverture de $4 \%$. Le constat est le même pour les Bouchesdu-Rhône ou le Bas-Rhin. À l'inverse, on observe la création de places dans des départements où on ne les aurait sans doute pas attendues au vu de la demande. Ainsi, l'Ouest français ressort largement sur cette carte, alors même qu'il ne faisait pas partie des départements de tête pour la demande d'asile (carte 1), ni des départements à forte 
tension entre hébergement et demande (carte 7). De même, la Lozère enregistre 16 demandes d'asile en 2003, possède 30 places d'hébergement spécifiques (toutes créées en 2003), pour un taux de couverture de $188 \%$. Les départements pour lesquels les nouvelles créations ont permis de réduire à la marge la tension sont très peu nombreux : Essonne, Seine-Maritime, Seine-et-Marne, Loiret, Hauts-de-Seine, Nord. Enfin, on observe des recompositions régionales intéressantes, qui tendraient vers une distribution de la demande d'asile vers les hébergements dans une logique de déconcentration régionale. Il est intéressant de noter que si le département de la Haute-Garonne (Toulouse) n'obtient pas de nouvelles places en 2003 alors que la situation est pour le moins tendue, ce sont les départements voisins du Gers et des Hautes-Pyrénées qui voient leurs capacités d'hébergement s'accroître. Il en est de même entre le Rhône (dont on peut signaler qu'il bénéficie seulement de 20 places CADA supplémentaires) et l'Ardèche (40 places).

Le croisement entre demandes d'asile et hébergement offre une autre géographie de la demande d'asile. Plusieurs points peuvent être soulignés. Si la situation nationale est globalement déficitaire, d'importantes nuances départementales apparaissent. Par ailleurs, le constat de l'absence de corrélation positive entre forte demande d'asile et fort taux d'encadrement en structures d'hébergement est immédiat. La logique de répartition des hébergements relève avant tout d'une logique d'équipement de l'ensemble du territoire français, ce qui ne saurait être le cas des logiques migratoires préférentielles des demandes d'asile. À un niveau plus fin, la situation du département du Rhône apparait intéressante. En effet, le Rhône présente la spécificité d'être un département accueillant une forte proportion de demandeurs d'asile, mais possédant également une «bonne " capacité d'hébergement relative, toutes proportions gardées sur l'adéquation impossible entre demandeurs et offres d'hébergement bien entendu. Cette situation relativement bonne, au regard de la moyenne nationale, demande un éclairage approfondi. Nous émettons cependant dès à présent l'hypothèse d'un rôle central joué par les acteurs locaux, qu'ils soient associatifs ou institutionnels.

\section{Les non-dits de la représentation cartographique}

31 Mais la carte ne dit pas tout, d'une part par absence et limite des données disponibles, d'autre part par limite de la représentation cartographique.

\section{Le manque de données disponibles}

Concernant l'analyse de l'évolution de la demande d'asile, les données départementales disponibles auprès de l'OFPRA ne remontent pas avant 1993. Le temps long ne peut donc pas être interrogé. Il conviendrait ici de remonter au moins jusqu'à l'année 1973, qui correspond à l'arrivée massive des demandeurs d'asile chiliens persécutés par la dictature de Pinochet, pour prendre en compte ensuite le phénomène des boat-people du sud-est asiatique.

Les données de l'OFPRA prennent uniquement en compte les personnes pour lesquelles un certificat de dépôt de demande d'asile a été délivré. Le schéma du début de la procédure (encadré 1) montre que ce certificat de dépôt intervient après plusieurs étapes dédiées à la régularisation du séjour des personnes en procédure. Les données statistiques relatives à ces étapes pilotées par les préfectures ne sont pas centralisées 
par l'OFPRA, ni par le Ministère de l'Intérieur en général, ou tout du moins elles ne sont pas diffusées, ce qui nuit à une analyse longitudinale de la procédure. De plus, les pratiques préfectorales sont diverses, notamment en terme de délai. Certaines préfectures accordent l'APS (Autorisation Provisoire de Séjour) sans délai, d'autres, surchargées, repoussent à plusieurs semaines, voire à plusieurs mois, l'établissement du titre provisoire et donc la remise du dossier qui devra être envoyé à l'OFPRA. Les personnes se trouvent alors dans une "zone grise » juridique, avec pour seul titre de séjour une convocation à la préfecture.

Pour les départements concernés par ces délais, les données de l'OFPRA comptabilisent donc le flux de demande avec retard. Il y a une déperdition entre le premier passage en préfecture et le certificat de dépôt. Ces déperditions sont difficiles à expliquer précisément. Elles sont liées sans doute pour partie à des abandons de procédure par découragement, mais aussi à des stratégies de maintien sur le territoire sans passage par l'APS, c'est-à-dire sans enregistrement informatisé des empreintes digitales. Inconnu de la base de données européenne (EURODAC), le demandeur peut alors se maintenir sur le territoire au moyen de premières demandes successives, sous diverses identités et/ou dans plusieurs départements.

Ces mobilités ne sont pas captées par les données de l'OFPRA, qui sous-estiment donc le nombre de premières demandes d'asile. Les cartes donnent une vision figée de ces premières demandes alors que le phénomène est spatialement très turbulent.

Concernant l'hébergement, les données disponibles ne permettent qu'une vision partielle de la réalité. En effet, il n'existe pas au niveau national d'évaluation précise de l'ensemble des dispositifs d'accueil des demandeurs d'asile (CADA, AUDA, CHRS, ALT, hôtels...) ni de données statistiques précises sur la situation des demandeurs d'asile qui ne bénéficient pas d'un hébergement dans le cadre du dispositif spécialisé et que l'on retrouve dans les foyers de travailleurs migrants, ou dans les hébergements relevant de l'urgence sociale et du caritatif. Les pouvoirs publics disposent seulement de données qualitatives éparses qui mettent en avant nombre de difficultés, notamment l'insuffisance des capacités d'accueil, l'incohérence de certaines situations locales, la difficulté d'accompagnement de ces populations, etc. Pour l'instant, c'est surtout à travers l'importance prise par la multiplication des cas d'hébergement d'urgence que cette réalité peut être appréhendée.

Partant de ces constats, la DREES (Direction de la Recherche des Études de l'Évaluation et des Statistiques - Ministère des affaires sociales et de l'emploi) a lancé fin 2002 un appel d'offres d'études visant à améliorer la connaissance du dispositif d'accueil et des populations elles-mêmes. Considérant que c'est au niveau départemental qu'apparaissent le mieux les pressions exercées par les demandeurs d'asile en matière d'hébergement, la DREES a retenu deux propositions: l'une conduite dans le département du Rhône (Bourgeois, Ebermeyer, Sévin, 2004) et l'autre dans les AlpesMaritimes (Frigoli, Jannot, 2004). Ces études avaient un caractère exploratoire puisqu'il s'agissait d'expérimenter une méthodologie d'approche du dispositif et des populations accueillies pour établir la faisabilité d'une enquête nationale. Finalement, l'enquête nationale paraît peu réalisable cette population étant difficile à connaître car particulièrement dispersée entre différents types de logements (structures spécialisées d'urgence, compatriotes, squat, rue,...). L'utilité d'une telle enquête est pourtant indiscutable. Il n'en demeure pas moins que les résultats de ces études fournissent, à l'échelle du département, quantité d'informations qui peuvent être utilement 
exploitées, notamment pour une représentation cartographique du croisement entre demande d'asile et structure d'hébergement. Ce sera l'objet d'un travail ultérieur qui sera conduit à l'échelle de l'agglomération lyonnaise.

\section{Quelques limites de la représentation cartographique}

La représentation cartographique occulte également une réalité difficile à appréhender, celle de la trajectoire résidentielle du demandeur d'asile sur le sol français. En effet, durant l'examen de son dossier (qui peut s'étaler sur plusieurs mois), le demandeur d'asile peut être amené à se déplacer, soit d'un département à un autre, soit au sein du même département, d'un lieu d'hébergement à un autre. Or, il est impossible, à partir des seules sources statistiques, de suivre et de reconstituer de tels parcours.

L'étude menée dans le département du Rhône a mis en évidence que seules des enquêtes auprès des populations concernées permettent, avec certaines réserves, de saisir cette réalité. Les constats généraux qui ont été établis à ce sujet sont les suivants : les parcours résidentiels des demandeurs d'asile sont caractérisés par l'instabilité ; ils sont marqués, qu'ils s'agissent de célibataires ou de familles avec enfants, par des périodes pendant lesquelles ils sont contraints de vivre dans la rue; ils sont différents entre familles et célibataires, les familles accédant plus facilement à un hébergement spécialisé. Néanmoins, les célibataires comme les familles connaissent des parcours résidentiels non linéaires, avec une succession de lieux de résidence, plus ou moins nombreux selon la durée de la procédure. En outre, la situation des demandeurs d'asile face à l'hébergement pose de nombreux problèmes, notamment celui de l'inégalité dans l'accès aux droits. Chacun sait qu'une personne hébergée en CADA aura plus de chances d'obtenir le statut de réfugié qu'une personne hébergée à l'hôtel, car celle-ci ne bénéficie d'aucun accompagnement dans sa procédure d'asile. L'accès à l'accompagnement pour obtenir le statut de réfugié est ainsi grandement déterminé par l'hébergement, d'où l'importance politique de cette question.

Cette réalité est, on l'aura compris, fort difficile à représenter cartographiquement, à moins de disposer d'une base de données suffisamment conséquente pour qu'apparaissent des typologies de trajectoires. À notre connaissance, un tel outil n'existe pas et reste à construire.

\section{BIBLIOGRAPHIE}

BRACHET Olivier(1997) L'impossible organigramme de l'asile en France : le développement de l'asile au noir, Revue Européenne des Migrations Internationales, vol. 13, n 1, pp. 7-36.

BOURGEOIS Frédérique, EBERMEYER Sophie, SEVIN Mélanie (2004) Demandeurs d'asile, réfugiés statutaires et déboutés dans les dispositifs d'accueil : étude exploratoire dans le département du Rhône, Lyon, Étude Économie \& Humanisme/Forum réfugiés pour la DREES, $163 \mathrm{p}$. 
BRACHET Olivier (2002) La condition du réfugié dans la tourmente de la politique d'asile, Hommes et Migrations, 1238, pp. 45-58.

DIRECTION DE LA POPULATION ET DES MIGRATIONS (2003) Rapport d'activité 2002, Paris, Ministère de l'Intérieur, $62 \mathrm{p}$.

FORUM RÉFUGIÉS (2003) Asile en France et perspectives européennes, Rapport 2003, Lyon, 137 p.

FRIGOLI Gilles, JANNOT Jessica (2004) L'hébergement des demandeurs d'asile comme enjeu local : éléments d'analyse et perspectives méthodologiques à partir du cas des Alpes-Maritimes, Nice, Étude URMIS pour la DREES, $199 \mathrm{p}$.

LEGOUX Luc (1993) La demande d'asile en France : le pic de 1989 et la théorie de la dissuasion, Revue Européenne des Migrations Internationales, vol. 9, n², pp. 31-41.

OFFICE FRANÇAIS DE PROTECTION DES RÉFUGIÉS ET DES APATRIDES (2004) Rapport d'activités 2003, Paris, Ministère des Affaires Étrangères, 28 p. + annexes.

\section{NOTES}

1. "Le terme de réfugié s'appliquera à toute personne (...) qui, (...) craignant avec raison d'être persécutée $d u$ fait de sa race, de sa religion, de sa nationalité, de son appartenance à un certain groupe social ou de ses opinions politiques, se trouve hors du pays dont elle a la nationalité et qui ne peut ou, du fait de cette crainte, ne veut se réclamer de la protection de ce pays ». Article 1A.3 de la Convention de Genève.

2. Les rapports d'activité de l'OFPRA sont disponibles gratuitement sur le site http:// www.ofpra.gouv.fr

3. Comme en témoigne le récent Géoforum de l'Association Française pour le Développement de la Géographie qui s'est tenu à Poitiers les 11 et 12 juin 2004 sur le thème des "Migrations internationales : connaître et comprendre ».

4. Ainsi la table thématique de la Revue Européenne des Migrations Internationales pour la période 1985-1998 ne mentionne que 17 articles portant sur le thème « réfugiés/demandeurs d'asile » sur un total de 672 références thématiques.

5. La loi du 10 décembre 2003 modifiant la loi relative à l'asile a supprimé la procédure de l'asile territorial à compter du $1^{\mathrm{er}}$ janvier 2004.

6. Depuis le mois de mai 2002 L'OFPRA enregistre dans sa base informatique les enfants mineurs accompagnant les demandeurs d'asile à titre personnel en tant que tels. Ainsi pour l'année 2003, l'OFPRA recense 7564 premières demandes de mineurs accompagnants. Ces données ne sont pas utilisées par la base départementale.

7. Voir dans ce numéro l'article de Gilles Frigoli.

\section{RÉSUMÉS}

Cet article propose un travail de représentation cartographique de la demande d'asile en France à partir du traitement des données de l'OFPRA. La traduction cartographique des données spatialisées relatives à la demande d'asile offre une première géographie de la demande d'asile en France, entre zones de forte densité et zones potentiellement moins chargées. Elle propose une 
première présentation des tendances nationales, revient sur leurs expressions départementales et interroge les réalités locales. La première partie s'intéresse à la distribution départementale des demandes d'asile en 2003, puis dans une approche dynamique en comparant temps moyen (1993-2003) et temps court (2002-2003), relevant ainsi les aléas des fluctuations inter-annuelles. La seconde partie de l'article croise la demande d'asile avec les capacités d'hébergement, elle montre ainsi la disjonction des deux données. Enfin, l'article met en relief la portée et les limites de la représentation cartographique du phénomène.

Mapping Out Requests for Asylum in France. National Trends, Departmental and Local Realities. This article puts forth a cartographic representation of requests for asylum in France based on analysis of data collected by the OFPRA (Office Français de Protection des Réfugiés et Apatrides: French Office for the Protection of Refugees and Stateless People). The cartographic translation of spatial data relating to requests for asylum offers an initial geography of the requests for asylum in France, between zones of high density and lower density. It provides a presentation of national trends, examines how these are expressed throughout the departments and inquires about local realities. The first part of the article looks into the distribution of asylum requests among departments in 2003, then uses a dynamic approach to compare the medium (1993-2003) and short-terms (2002-2003) to point out year-to-year fluctuations. The second part contrasts requests for asylum with housing capacity, showing the disjunction between the two. Lastly, the article highlights the significance and the limits of cartographic representation of this phenomenon.

Cartografiar la demanda de asilo en Francia. Tendencias nacionales, representaciones provinciales y realidades locales. Este artículo propone un trabajo de representación cartográfica sobre la demanda de asilo en Francia según el proceso de datos de la Delegación Francesa de Protección de Refugiados y Apátridas. La traducción cartográfica de los datos espacializados sobre la demanda de asilo en Francia muestra una primera representación geográfica entre zonas de fuerte demanda y zonas donde dicha demanda es menor. Propone una primera presentación de las tendencias nacionales, vuelve sobre sus expresiones provinciales y analiza las realidades locales. La primera parte se interesa por la distribución provincial de la demanda de asilo en el 2003, para luego, con un enfoque dinámico, comparar entre un intervalo medio (1993-2003) y uno corto (2002-2003), poniendo de relieve, de esta manera, los avatares de las fluctuaciones entre los años. La segunda parte del artículo cruza la demanda de asilo con las capacidades de alojamiento mostrando así la disyunción de los dos datos. Por último, el artículo pone de relieve el alcance y los límites de la representación cartográfica del fenómeno.

INDEX

Index géographique : France

Mots-clés : cartographie, demandeurs d'asile, méthodologie, répartition géographique

\section{AUTEURS}

\section{EMMANUELLE BONERANDI}

Maître de conférences en géographie, UMR Géographie-Cités - Géophile, ENS Lettres et Sciences Humaines, 15 parvis René Descartes, BP 7000, 69342 Lyon cedex, ebonerandi@ens-lsh.fr 


\section{FRÉDÉRIQUE BOURGEOIS}

Adjointe de direction, Forum Réfugiés, Siège Social, BP 1054, 69612 Villeurbanne cedex, fbourgeois@forumrefugies.org

\section{XAVIER RICHARD}

Chargé d'opérations Domiciliation, Forum Réfugiés, 82 quai Perrache 69002 Lyon, xrichard@forumrefugies.org 